\title{
The Critical Exponent for A Class Non-Autonomous Parabolic Equation in the K-Times Halved Space
}

\section{Youhua Peng ${ }^{1, a^{*}}$ Xianfeng Huang ${ }^{2, b}$}

${ }^{1}$ College of Engineering and Management, Pingxiang University, Pingxiang Jiangxi 337055, China ${ }^{2}$ Department of Mathematics, Hunan Normal University, Changsha Hunan 410081, China a*pengyouh@126.com, bemail,

Keywords: Critical Exponent, Non-Autonomous Parabolic Equation, K-Times Halved Space

Abstract: This paper investigates the parabolic equation

$$
u_{t}=\Delta u+t^{\sigma}|x|^{m} u^{p},(x, t) \in D \times(0, T) \quad\left(P^{*}\right)
$$

with

nonnegative

initial

date, where $D=R_{K}^{N}:=\left\{x \in R^{N} \mid x_{i}>0, i=1,2, \cdots K\right\}, 0 \leq K \leq N, \sigma \geq 0, m>-2, p>1$ and extend the classical result of Fujita and more recent results of Levine and Meier. We demonstrate that $p_{c}=1+\frac{2+2 \sigma+m}{N+K}$ as its critical exponent, which means that problem ( $P^{*}$ ) exhibited the following behavior: if $1<p<p_{c}$, then every positive solution of the equation blow up in finite time, whereas if $p>p_{c}$, then there exist both global and nonglobal solutions.

\section{Introduction and the Main Results}

In this paper, we will consider existence and nonexistence of the following problem

$$
\left\{\begin{array}{cc}
u_{t}=\Delta u+t^{\sigma}|x|^{m} u^{p} & (x, t) \in D \times(0, T) \\
u(x, t)=0 & x \in \partial D \times(0, T) \\
u(x, 0)=u_{0}(x) \geq 0 & x \in D
\end{array}\right.
$$

Where $\sigma \geq 0, m>-2, p>1, D=R_{K}^{N}:=\left\{x \in R^{N} \mid x_{i}>0, i=1,2, \cdots K\right\}, 0 \leq K \leq N$, and $u_{0}(x)$ is a nonnegative, continuous and bounded function.

The study of blow-up for nonlinear parabolic equation originates from Fujita[1]. He considered the following cauchy problem of the semilinear heat equation

$$
\left\{\begin{array}{cc}
u_{t}=\Delta u+u^{p} & (x, t) \in R^{N} \times(0, T) \\
u(x, 0)=u_{0}(x) \geq 0 & x \in R^{N}
\end{array}\right.
$$

He proved the following result:

(a) If $1<p<1+2 / N$,then (1.2) possesses no global nonnegative solutions.

(b) If $p>1+2 / N$, both global and nonglobal nonnegative solutions exist.

The case $p=1+2 / N$ belongs to case (a), but this was established later (see[5] Weissler give 
an elegant proof). We call $p_{c}=1+2 / N$ the critical exponent of (1.2).

Over the past few years, there have been a number of extensions of Fujita's results in various directions(see for example [1]-[13]). Levine [2] treated the following equation

$$
\left\{\begin{array}{lc}
u_{t}=\Delta u+t^{\sigma}|x|^{m} u^{p} & (x, t) \in R^{N} \times(0, T) \\
u(x, 0)=u_{0}(x) \geq 0 & x \in R^{N}
\end{array}\right.
$$

he showed the critical exponent of (1.3) is $p_{c}=1+\frac{2+2 \sigma+m}{N}$.

For the domain $D=R_{K}^{N}$, Meier [6] studied the boundary-value problem

$$
\left\{\begin{array}{cc}
u_{t}=\Delta u+t^{\sigma} u^{p} & (x, t) \in D \times(0, T) \\
u(x, t)=0 & x \in \partial D \times(0, T) \\
u(x, 0)=u_{0}(x) \geq 0 & x \in D
\end{array}\right.
$$

He proved the critical exponent of (1.4) is $p_{c}=1+\frac{2+2 \sigma}{N+K}$.

We are concerned problem (1.1) and our Fujita's type results are contained in the following two theorems.

Theorem 1. Suppose that $\sigma \geq 0, m>-2, p>1,0 \leq K \leq N$, and $u_{0}(x)$ is a nonnegative, continuous and bounded function. If $1<p<1+\frac{2+2 \sigma+m}{N+K}$, then every nonnegative solution of (1.1) blow up in finite time.

Theorem 2. Suppose that $\sigma \geq 0, m>-2, p>1,0 \leq K \leq N$, and $u_{0}(x)$ is a nonnegative, continuous and bounded function. If $p>1+\frac{2+2 \sigma+m}{N+K}$, then

(a) Every nonnegative solution of (1.1) blow up in finite time for $u_{0}(x)$ large enough.

(b) (1.1) has global solutions for $u_{0}(x)$ small enough.

The method that we used is similar to the method used to prove the blow-up result by Pinsky in [8] . So we need the Green's function of the heat equation in the K-times halved space D (see [7]). That is

$$
G(x, y, t)=(4 \pi t)^{-\frac{N}{2}}\left(\prod_{i=1}^{K} D\left(x_{i}, y_{i}, t\right)\right)\left(\prod_{j=k+1}^{N} E\left(x_{i}, y_{i}, t\right)\right)
$$

where $E(x, y, t)=\exp \left(-\frac{|x-y|^{2}}{4 t}\right)$ and $D(x, y, t)=E(x, y, t)-E(x,-y, t)$.

Throughout the rest of this paper, we will use the notation

$$
y=\left(y_{1}, \cdots, y_{K}, y_{K+1}, \cdots y_{N}\right), \bar{y}=\left(-y_{1}, \cdots,-y_{K}, y_{K+1}, \cdots y_{N}\right)
$$

This paper is organized as follows: In section 2, we will prove some preliminary results. The proofs of the main results are completed in section 3. 


\section{Preliminary}

In this section, we give some lemmas needed in the proofs of Theorem 1 and Theorem 2.

Lemma 2.1 Assume $G(x, y, t)$ is the Green's function of the heat equation in the K-times halved space D. Then

$$
G(x, y, t) \geq 2^{-\frac{N}{2}} t^{-\frac{N}{2}-K} E\left(0, \bar{y}, \frac{t}{2}\right) \exp \left(-\frac{|x|^{2}}{2 t}\right)\left(\prod_{i=1}^{K} x_{i} y_{i}\right)
$$

Proof. By an elegant calculation, we show $D\left(x_{i}, y_{i}, t\right)=\exp \left(-\frac{x_{i}^{2}+y_{i}^{2}}{4 t}\right)\left[\exp \left(\frac{x_{i} y_{i}}{2 t}\right)-\exp \left(-\frac{x_{i} y_{i}}{2 t}\right)\right]$ Applying the Mean Value Theorem, it follows that

$$
D\left(x_{i}, y_{i}, t\right) \geq t^{-1} \exp \left(-\frac{x_{i}^{2}+y_{i}^{2}}{4 t}\right) x_{i} y_{i}
$$

Thus from (2.2), we obtain

$$
G(x, y, t) \geq(4 \pi t)^{-\frac{N}{2}} t^{-K} \exp \left(-\frac{(x-\bar{y})^{2}}{4 t}\right)\left(\prod_{i=1}^{K} x_{i} y_{i}\right)
$$

Using the inequality $|x-y|^{2} \leq 2|x|^{2}+2|y|^{2}$; we complete the proof .

Lemma 2.2 Suppose $u(x, t)$ satisfies (1.1). Then there exist $t_{0}, c_{1}>0$ such that

$$
u(x, t) \geq c_{1} t^{-\frac{N}{2}-K} \exp \left(-\frac{|x|^{2}}{2 t}\right)\left(\prod_{i=1}^{K} x_{i}\right) \text { for any } t>t_{0} .
$$

Proof. Using Lemma 1 of [8], we can similarly prove that

$$
u(x, t) \geq \int_{D} G(x, y, t) u_{0}(y) d y+\int_{0}^{t} \int_{D} G(x, y, t-s)|y|^{m} s^{\sigma} u^{p}(y, s) d y d s
$$

Since $u_{0}(x) \geq 0$, it follows from (2.5) that

$$
u(x, t) \geq \varepsilon \int_{B_{\varepsilon}\left(y_{0}\right)} G(x, y, t) d y \quad \text { for some } \varepsilon>0 \text { and some } y_{0} \in D
$$

where $B_{\varepsilon}\left(y_{0}\right)$ denotes the ball of radius $\varepsilon>0$ centered at $y_{0}$.

By Lemma (2.1), we complete the proof .

Lemma 2.3 Suppose $u(x, t)$ satisfies (1.1) for all $\mathrm{T}>0$ and that

$$
u(x, t) \geq c t^{l} \exp \left(-\frac{|x|^{2}}{t}\right)\left(\prod_{i=1}^{K} x_{i}\right) \quad \text { for } t>t_{0}
$$

where $c>0, t_{0}>0$, and $l \in\left[-\frac{N}{2}-K,+\infty\right]$. Then there exist constants $C, t_{1}>0$, such that

$$
u(x, t) \geq C t^{l+\sigma+l p+\frac{m+(p-1) K}{2}} \exp \left(-\frac{|x|^{2}}{t}\right)\left(\prod_{i=1}^{K} x_{i}\right), \quad \text { for } t>t_{1}
$$


Proof. From (2.5) and (2.8), we have

$$
u(x, t) \geq c^{p} \int_{t_{0}}^{t} \int_{D} G(x, y, t-s)|y|^{m} s^{\sigma+l p} \exp \left(-\frac{p|y|^{2}}{t}\right)\left(\prod_{i=1}^{K} y_{i}^{p}\right) d y d s
$$

Using the equality $\exp \left(-\frac{\left(y_{i}-x_{i}\right)^{2}}{4(t-s)}-\frac{p y_{i}^{2}}{s}\right)=\exp \left(-\frac{\left(y_{i}-r(s, t) x_{i}\right)^{2}}{4 r(s, t)(t-s)}\right) \exp \left(-\frac{p r(s, t) x_{i}^{2}}{s}\right)$

Where $r(s, t)=\frac{s}{s+4 p(t-s)}$.

We obtain $D\left(x_{i}, y_{i}, t-s\right) \exp \left(-\frac{p y_{i}^{2}}{s}\right)=D\left(r(s, t) x_{i}, y_{i}, t-s\right) \exp \left(-\frac{p r(s, t) x_{i}^{2}}{s}\right)$

And $G(x, y, t-s) \exp \left(-\frac{p y^{2}}{s}\right) \geq 2^{-\frac{N}{2}}(t-s)^{-\frac{N}{2}-K}\left(\prod_{i=1}^{K} x_{i} y_{i}\right) E(0, \bar{y}, r(s, t)(t-s) / 2)$

$$
\times \exp \left(-\frac{r(s, t)|x|^{2}}{2(t-s)}\right) \exp \left(-\frac{\operatorname{pr}(s, t)|x|^{2}}{s}\right)
$$

Clearly $(1+r(s, t))(t /(t-s))<4$, for $s \in[0, t / 2]$ So we have

$$
\exp \left(-\frac{r(s, t)|x|^{2}}{2(t-s)}\right) \exp \left(-\frac{\operatorname{pr}(s, t)|x|^{2}}{s}\right)=\exp \left(-\frac{|x|^{2}}{4 t}(1+r(s, t))(t /(t-s))\right) \geq \exp \left(-\frac{|x|^{2}}{t}\right)
$$

Substituting (2.11), (2.12) into (2.10), we conclude

$$
u(x, t)=c^{p} 2^{-\frac{N}{2}} \exp \left(-\frac{|x|^{2}}{t}\right)\left(\prod_{i=1}^{K} x_{i}\right) \int_{t_{0}}^{t / 2} \int_{D} E(0, \bar{y}, r(s, t)(t-s) / 2)(t-s)^{-\frac{N}{2}-K}\left(\prod_{i=1}^{K} y_{i}^{p+1}\right)|y|^{m} s^{\sigma+l p} d y d s
$$

Noticing that there exists constant $c_{2}>0$ such that

$$
\int_{D}(4 \pi t)^{-\frac{N}{2}} E(0, \bar{y}, t)\left(\prod_{i=1}^{K} y_{i}^{p+1}\right)|y|^{m} d y \geq c_{2} t^{\frac{m+(p+1) K}{2}} \text {, for } t \geq 1 \text {. }
$$

We show $\int_{D}\left(r(s, t)(t-s)^{-\frac{N}{2}} E(0, \bar{y}, r(s, t)(t-s) / 2)\left(\prod_{i=1}^{K} y_{i}^{p+1}\right)|y|^{m} d y \geq c_{3}\left(r(s, t)(t-s)^{\frac{m+(p+1) K}{2}}\right.\right.$

where $c_{3}$ is a positive constant, $r(s, t)(t-s) / 2 \geq 1$.

An elegant calculation reveals that for sufficiently large $h \geq 1$, $r(s, t)(t-s) / 2 \geq 1$, if $t \geq 2 h$ and $h \leq s \leq t-h$

Without loss of generality, we suppose that $h \geq t_{0}$. From (2.13) and (2.15), it follows that there exists constant $c_{4}>0$ such that

$$
u(x, t)=c_{4} \exp \left(-\frac{|x|^{2}}{t}\right)\left(\prod_{i=1}^{K} x_{i}\right) \int_{h}^{t / 2} \int_{D} s^{\sigma+l p}(r(s, t))^{\frac{N+m+(p+1) K}{2}}(t-s)^{\frac{m+(p-1) K}{2}} d s \text {, for } t \geq 2 h .
$$


Making the change of variables $z=s / t,(2.16)$ can be rewritten as

$$
\begin{gathered}
u(x, t)=c_{4} \exp \left(-\frac{|x|^{2}}{t}\right)\left(\prod_{i=1}^{K} x_{i}\right) t^{1+\sigma+l p+\frac{m+(p-1) K}{2}} \\
\int_{h}^{t / 2} s^{\sigma+l p}\left(\frac{z}{z+4 p(1-z)}\right)^{\frac{N+m+(p+1) K}{2}}(1-z)^{\frac{m+(p-1) K}{2}} d z \text {, for } t \geq 2 h .
\end{gathered}
$$

If we now assume that $t \geq 4 h$, from (2.17), we assert that there exist constants $C, t_{1} \geq 0$ such that $u(x, t) \geq C t^{l+\sigma+l p+\frac{m+(p-1) K}{2}} \exp \left(-\frac{|x|^{2}}{t}\right)\left(\prod_{i=1}^{K} x_{i}\right), \quad$ for $t>t_{1}$.

Lemma 2.4 Suppose $u(x, t)$ satisfies (1.1), $\Omega$ is bounded subset of D. If $1<p<1+\frac{2+2 \sigma+m}{N+K}$, Then $\lim _{t \rightarrow+\infty} u(x, t)=+\infty, x \in \Omega$.

Proof. Define the affine transformation $f(l)=1+\sigma+l p+\frac{m+(p-1) K}{2}$, for $l \in R$. We find that $f^{n}\left(-\frac{N}{2}-K\right)=\frac{1}{p-1}\left[\frac{2+2 \sigma+m-(p-1)(N+K)}{2} p^{n}-\left(\frac{2+2 \sigma+m}{2}+\frac{K p}{2}-\frac{K}{2}\right)\right]$. The condition $1<p<1+\frac{2+2 \sigma+m}{N+K}$ guarantees that $2+2 \sigma+m-(p-1)(N+K)>0$. then we obtain $\lim _{t \rightarrow+\infty} f^{n}\left(-\frac{N}{2}-K\right)=+\infty$.

By Lemma (2.2), (2.8) holds with $l=-\frac{N}{2}-K$, after applying $n$ iterations of Lemma (2.3),

$$
\text { we have } u(x, t)=C t^{f^{n}\left(-\frac{N}{2}-K\right)} \exp \left(-\frac{|x|^{2}}{t}\right)\left(\prod_{i=1}^{K} x_{i}\right) \text {, for } t \geq t_{n} \text {. }
$$

Now (2.18) follows from (2.19) and (2.20).

\section{The Proofs of Theorem 1 and Theorem 2}

Proof of Theorem 1: Suppose that the conclusion of Theorem 1 is false. That is, when $1<p<1+\frac{2+2 \sigma+m}{N+K}$, (1.1) possesses global solutions. By the Lemma (2.4), we shift (if necessary) the origin of time to $\bar{t}$ such that $u(x, \bar{t})=u_{0}^{\prime}(x)$ is large enough, where $\bar{t}$ is a nonnegative constant. Let $\Omega \in D$ be a smooth, bounded domain satisfying $\inf _{x \in \Omega}|x|^{m} \geq \delta$. We consider the following boundary value problem

$$
\left\{\begin{array}{cc}
U_{t}=\Delta U+\delta \bar{t}^{-\sigma} U^{p} & (x, t) \in \Omega \times(\bar{t}, T) \\
U(x, t)=0 & x \in \partial \Omega \times \bar{t}, T) \\
U(x, 0)=u_{0}^{\prime}(x) \geq 0 & x \in \Omega
\end{array}\right.
$$


By The Comparison Theorem, we obtain $u(x, t) \geq U(x, t),(x, t) \in \Omega \times(\bar{t}, T)$.

Clearly, when $u_{0}^{\prime}(x)$ is large enough, $U(x, t)$ will blow up in finite time. It follows from (3.2) that $u(x, t)$ will blow up in finite time. This contradicts to the supposition that (1.1) possesses global solutions.

Proof of Theorem 2: Part (a) is obvious. Since $u_{0}(x)$ is large enough, the proof is almost the same as the proof of Theorem 1 .

Now we prove part (b). We look for a supersolution of (1.1). Take

$$
\bar{u}(x, t)=A(T+t)^{-\alpha} e^{-\omega|\eta|^{2}}\left(\prod_{i=1}^{K} x_{i}\right) \text {, where } \eta=\frac{x}{\sqrt{T-t}} .
$$

By an elegant calculation, $\bar{u}(x, t)$ need satisfy

$$
-\alpha+2 \omega N+4 \omega K \geq \omega(4 \omega-1)|\eta|^{2}+A^{p-1}(T+t)^{1+\alpha-\alpha p} t^{\sigma}|x|^{m} e^{(1-p) \omega|\eta|^{2}}\left(\prod_{i=1}^{K} x_{i}\right)^{p-1}
$$

Since $p>1$, there exists a nonnegative constant $C$ such that

$$
t^{\sigma}|x|^{m} e^{(1-p) \omega|\eta|^{2}}\left(\prod_{i=1}^{K} x_{i}\right) \leq C(T+t)^{\sigma+\frac{m+(p-1) K}{2}}
$$

Choose $\omega=1 / 4,(3.4)$ is satisfied if $-\alpha+\frac{N}{2}+K \geq C A^{p-1}(T+t)^{1+\alpha-\alpha p+\sigma+\frac{m+(p-1) K}{2}}$

Let $1+\alpha-\alpha p+\sigma+\frac{m+(p-1) K}{2}=0$. Then $\alpha=\frac{2+2 \sigma+m}{2 p-2}+\frac{K}{2}$. Since $p>1+\frac{2+2 \sigma+m}{N+K}$.

So (3.6) holds when $A$ is small enough. Thus, we complete the proof of part (b).

\section{Acknowledgements}

Fund Project: National Natural Science Foundation (11661065), the Jiangxi Provincial Department of Education 2015 Annual Scientific And Technological Research Projects (GJJ151274).

\section{References}

[1] H. Fujita, On the blowing up of solutions of the Cauchy problem for $u_{t}=\Delta u+u^{1+\alpha}$, J. Fac. Sci. Uni. Tokyo Sec. 1 A Math. 16 (1966), 105-113.

[2] H. Levine, The Role of Critical Exponents in Blowup Theorems. SIAM Review. 32 (1990), 262-288.

[3] H. A. Levine and P. Meier, A blowup result for the critical exponent in cones, Israel J. Math., 67(1989), 1-7.

[4] H. A. Levine and P. Meier, The value of the critical exponent for reaction-diffusion equations in cones, Arch. Rational Mech. Anal. 1091990, 73-80.

[5] F. B. Weissler, Existence and nonexistence of global solutions for a semilinear heat equation, Israel J. Math. 38(1981), 29-40. 
[6] P. Meier, Blow-up of solutions of semilinear parabolic differential equations. Z. Angew. Math. Phys., 39(1988), 135-149.

[7] P. Meier, Existence et non-existence de solutions globales d'une equation de la chaleur semi-lineaire: extension dun theoreme de Fujita , C. R. Acad. Sci, Paris Serie 1, 303 (1986),635-637.

[8] R. G. Pinsky, Existence and Nonexistence of Global Solutions for $u_{t}=\Delta u+a(x) u^{p}$ in $R^{d}$. J. D. E., 133(1997), 152-177.

[9] V. A. Galaktionov and H. A. Levine, On critical Fujita exponents for heat equations with nonlinear flux conditions on the boundary, Israel J. Math. 94 (1996), 125-146.

[10] V. A. Galaktionov and J. L. Vazquez, Continuation of blowup solutions of nonlinear heat equations in several space dimensions, Comm. Pure Appl. Math., 50 (1997), 1-67.

[11] T. Hamada, Nonexistence of global solutions of parabolic equation in conical domains, Tsukuba J. Math., 19 (1995), 15-25

[12] B. Hu, Remarks on the blowup estimate for solution of the heat equation with a non-linear boundary condition, Differential Integral Equations, 9(1996), 891-901.

[13] K. Kobayashi, T. Sirao and H. Tanaka, On the blowing up problem for semilinear heat equations, J. Math. Soc. Japan, 29 (1977), 407-424. 